\title{
Analysis and Optimization of Coupled Windings in Magnetic Resonant Wireless Power Transfer Systems with Orthogonal Experiment Method
}

\author{
Xiao Yudi ${ }^{1}$, Mao Xingkui ${ }^{1,2}$, Lin Mao $^{3}$, Zhang Zhe ${ }^{2}$, Michael A. E. Andersen ${ }^{2}$ \\ ${ }^{I}$ College of Electrical Engineering and Automation, Fuzhou University, \\ Fuzhou, China \\ ${ }^{2}$ Department of Electrical Engineering, Technical University of Denmark, \\ Kgs. Lyngby, Denmark \\ ${ }^{3}$ Gutian River Hydropower Plant, \\ Gutian County, China \\ contact.mxk782@fzu.edu.cn
}

\begin{abstract}
The coupled magnetic resonant unit (CMRU) has great effect on the transmitting power capability and efficiency of magnetic resonant wireless power transfer system. The key objective i.e. the efficiency coefficient $k_{Q}$ is introduced in the design of CMRU or the coupled windings based on the mutual inductance model. Then the design method with orthogonal experiments and finite element method simulation is proposed to maximize the $k_{\mathrm{Q}}$ due to low precise analytical model of $\mathrm{AC}$ resistance and inductance for $\mathrm{PCB}$ windings at high- frequency. The method can reduce the design iterations and thereby can get more optimal design results. The experiments verified the design objective of $k_{Q}$ as well as the design method effectively. In the optimal PCB windings prototype at operating frequency of 4 $\mathrm{MHz}$, the $k_{\mathrm{Q}}$ and the maximum efficiency are increased by about $12 \%$ and $4 \%$ respectively.
\end{abstract}

Index Terms-Wireless power transfer; magnetic resonant; orthogonal experiments; magnetic coupling structure optimization.

\section{INTRODUCTION}

Medium-range efficient power transfer can be implemented through magnetic resonant wireless power transfer (MR-WPT), where the coupled windings resonate to exchange energy efficiently [1]-[5]. The coupled magnetic resonant unit (CMRU) consisting of coupled windings and compensation capacitances, as one of the key components in MR-WPT, has great effects on the system transmitting power capability, efficiency, distance, stability, etc. In the modelling (including the coupled mode theory and the circuit theory [6], [7]) and design of CMRU, the coupled winding parameters, such as AC resistance $R_{\mathrm{ac}}$, inductance, or mutual inductance $M$ should be required. Under high-frequency operation, the $R_{\mathrm{ac}}$ and $M$ depends on the frequency greatly because of high-frequency eddy-current effect. There are some literatures [8]-[12] on the analytical modelling of the

Manuscript received 21 December, 2016; accepted 28 July, 2017.

This research was funded by a grant (Natural Science Foundation of China, No. 51207025, and of Fujian Province, China, No.2017J01689). This research was performed in cooperation with the Department of Electrical Engineering, Technical University of Denmark. transformer and inductor windings. In addition, they are available in the modelling and design of the coupled windings of CMRU [12]-[14]. But under higher operation frequency, such as several hundreds of $\mathrm{kHz}$ and $\mathrm{MHz}$, the analytical modelling will have poor accuracy, especially for the coupled PCB windings characterized of much more fringing high-frequency magnetic field comparing with the magnetic-core transformer and inductor. In order to achieve an enough or higher accuracy, finite element method (FEM) simulation would be preferred. However, it will result in increasing simulation time greatly as for the optimal design of the coupled windings, especially with the method of the scanning parameters being available in FEM software. The method with single parameter analysis and FEM simulation [15], in which the influence of varying one parameter was studied while fixing all the other parameters, is utilized to simplify analysis and optimal design of the coupled windings. However, it will perhaps lose the best optimal design because there are interactions among the parameters. Therefore a method with orthogonal experiments and FEM simulation is proposed, and it is able to make trade-offs between design time and optimal solutions. The orthogonal experiment is one of optimal design methods, where only the representative experiments are selected [16].

The paper investigates the design objective based on the mutual inductance firstly, then analyses the interactions among the parameters and designs the orthogonal experiments, finally makes optimal design and verification.

\section{Model of CMRU AND THE Design OBJECTIVE}

The CMRU can be classified into two types: two winding structure and four winding structure [2]. The mutual inductance model for the four winding structure with series-connecting compensation capacitance is shown in Fig. 1. In the model, $I_{S}$ and $R_{S}$ is the high-frequency exciting source and its internal resistance; $L_{1}, L_{2}, L_{3}$, and $L_{4}$ represent self-inductance of each winding respectively; $C_{1}, C_{2}, C_{3}$, and $C_{4}$ represent series-connecting capacitance for each winding; $R_{1}, R_{2}, R_{3}$, and $R_{4}$ represent the resistance of each winding 
under high-frequency current; $M_{12}, M_{23}$, and $M_{34}$ represents mutual inductance between the two adjacent windings; $R_{\mathrm{L}}$ is the load resistance; $Z_{\mathrm{i}}=R_{\mathrm{i}}+\mathrm{j} X_{\mathrm{i}}$ represents the input impedance of the coupled magnetic unit; $Z_{\mathrm{I}}^{\prime}=R_{\mathrm{I}}^{\prime}+\mathrm{j} X_{\mathrm{i}}^{\prime}$ represents impedance from the emitting winding port; $Z_{\mathrm{o}}=R_{\mathrm{o}}$ $+\mathrm{j} X_{\mathrm{o}}$ represents equivalent load impedance from the load winding port. The efficiency of the MR-WPS with the four winding structure is divided into three parts $\eta_{12}, \eta_{23}$, and $\eta_{34}$ as in Fig. 1. $\eta_{12}, \eta_{23}$, and $\eta_{34}$ are the transmitting efficiency between the source and the emitting winding, the emitting winding and the receiving winding, the receiving winding and the load winding respectively.

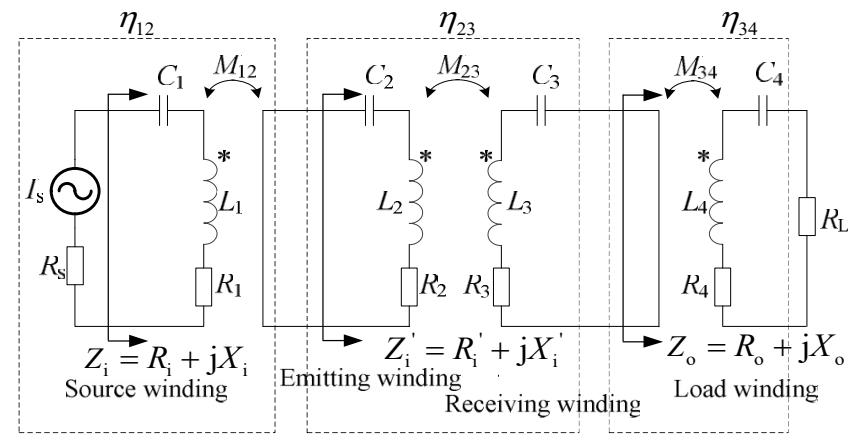

Fig. 1. Mutual inductance model of the four-winding structure.

In the design, both source compensation winding $L_{1}$ and load compensation winding $L_{4}$ are constructed with single turn to obtain small winding resistance $R_{1}$ and $R_{4}$. Impedances $Z_{\mathrm{i}}, Z_{\mathrm{i}}^{\prime}$ and $Z_{\mathrm{o}}$ is purely resistive when each winding operates resonantly by design of source frequency and compensation capacitances, thus $R_{\mathrm{i}}, R_{\mathrm{i}}^{\prime}$, and $R_{\mathrm{o}}$ can be written as follows:

$$
\left\{\begin{array}{l}
R_{\mathrm{i}}=R_{1}+\frac{\left(\omega_{0} M_{12}\right)^{2}}{R_{\mathrm{i}}^{\prime}} \approx \frac{\left(\omega_{0} M_{12}\right)^{2}}{R_{\mathrm{i}}^{\prime}}, \\
R_{\mathrm{i}}^{\prime}=R_{2}+\frac{\left(\omega_{0} M_{23}\right)^{2}}{R_{3}+R_{\mathrm{O}}} \\
R_{\mathrm{o}}=\frac{\left(\omega_{0} M_{34}\right)^{2}}{R_{4}+R_{\mathrm{L}}} \approx \frac{\left(\omega_{0} M_{34}\right)^{2}}{R_{\mathrm{L}}}
\end{array}\right.
$$

$\omega_{0}$ represents resonant frequency in the equation.

The total efficiency $\eta$ of the CMRU can be expressed as a product of transmission efficiency between the two adjacent windings i.e. $\eta=\eta_{12} \eta_{23} \eta_{34}$, where $\eta_{12} \approx R_{\mathrm{i}} /\left(R_{\mathrm{s}}+R_{\mathrm{i}}\right), \eta_{34} \approx 1$ because of small winding resistance $R_{4}$

$$
\eta_{23}=\frac{\left(\omega_{0} M_{23}\right)^{2} R_{\mathrm{o}}}{\left(R_{3}+R_{\mathrm{o}}\right)\left(R_{2}\left(R_{3}+R_{\mathrm{o}}\right)+\left(\omega_{0} M_{23}\right)^{2}\right)} .
$$

When the equivalent load equal to optimal equivalent load resistance, $R_{\mathrm{O} \_ \text {opt }}=\sqrt{R_{3}\left(\omega_{0} M_{23}\right)^{2} / R_{2}+R_{3}{ }^{2}}, \eta_{23}$ will be maximized as:

$$
\eta_{23 \_ \text {max }}=\frac{k_{\mathrm{Q}}^{2}}{k_{\mathrm{Q}}^{2}+2 \sqrt{k_{\mathrm{Q}}^{2}+1}+2},
$$

$$
k_{\mathrm{Q}}=k_{23} \sqrt{Q_{2} Q_{3}},
$$

where $k_{23}=M_{23} / \sqrt{L_{2} L_{3}}$ represents the coupling coefficient between the emitting winding and the receiving winding; $Q_{2}=\omega_{0} L_{2} / R_{2}$ and $Q_{3}=\omega_{0} L_{3} / R_{3} \mathrm{r}$ epresent the quality factor of the emitting and receiving windings respectively. It can be seen that, the maximum efficiency $\eta_{23}$ max only depends on $k_{\mathrm{Q}}$ determined by the intrinsic parameters $k_{23}, Q_{2}$, and $Q_{3}$ of the windings.

The curve of Fig. 2 can be plotted from (3). It can be seen that, $\eta_{23}$ max will decrease with decreasing $k_{\mathrm{Q}}$, and drops rapidly on the left side of the point a. Therefore, $k_{\mathrm{Q}}$ should be designed on the right side of point a if there are no limitations on selecting $k_{\mathrm{Q}}$ or the windings geometry. Moreover, if the $k_{\mathrm{Q}}$ has to be designed on the left side of point a due to some practical restrictions, it must be designed as large as possible for getting a high efficiency or high capability of power transmission. Here the $k_{\mathrm{Q}}$ is introduced to be as the design objective for the coupled windings.

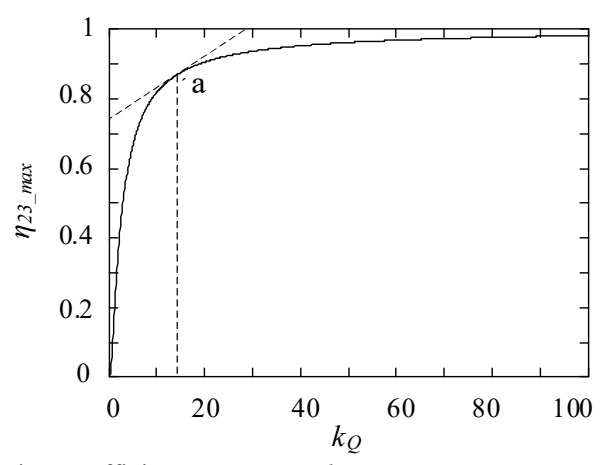

Fig. 2. Maximum efficiency $\eta_{23}{ }_{-} \max v$ vs. $k_{\mathrm{Q}}$.

Since $\eta_{23}$ only denotes transmitting efficiency between the emitting winding and the receiving winding, the two-winding coupling structure, instead of the four-winding coupling structure, is adopted to verify the relationship between the maximum efficiency $\eta_{23}$ max and $k_{\mathrm{Q}}$. As shown in Fig. 3 , three pairs of coupling windings are constructed. In each pair of the coupling windings, the emitting winding and the receiving winding have the same diameter, which is $40 \mathrm{~mm}, 50 \mathrm{~mm}$ and $63 \mathrm{~mm}$ respectively; Both emitting winding and receiving winding are solenoid windings with number of turns of 10 , solenoid length of $15 \mathrm{~mm}$ and wire diameter of $0.65 \mathrm{~mm}$. The exciting current frequency is $3.9 \mathrm{MHz}$.

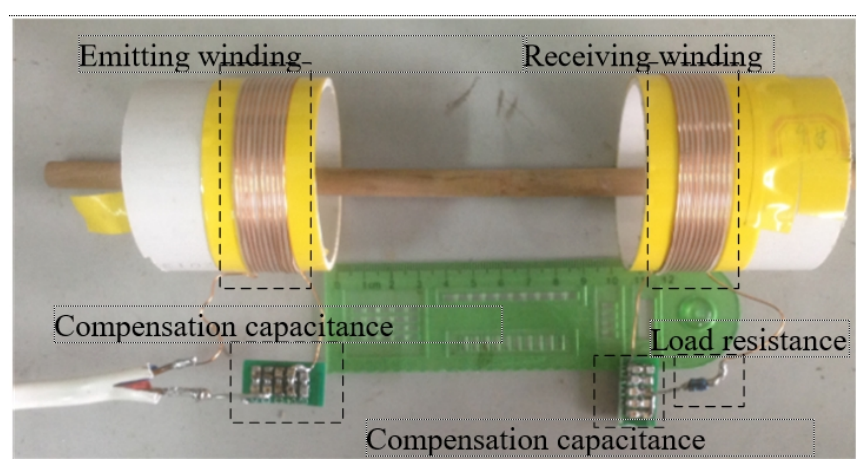

Fig. 3. Constructed windings for the model verification.

In order to compare the maximum efficiency of each pair under a same efficiency coefficient $k_{\mathrm{Q}}$, corresponding 
transmitting distance of the each pair for a same efficiency coefficient is calculated through (4) and the winding tested parameters listed in Table I (where $L$ and $R_{\text {ac }}$ represents self-inductance and equivalent $\mathrm{AC}$ resistance of the windings respectively, $C$ represents compensation capacitance). Table II shows the calculating transmitting distance for the same efficiency coefficient.

TABLE I. WINDING PARAMETERS OF EACH PAIR.

\begin{tabular}{|c|c|c|c|c|}
\hline & Diameter $(\mathbf{m m})$ & $\boldsymbol{L}(\boldsymbol{\mu} \mathbf{H})$ & $\boldsymbol{C}(\mathbf{p F})$ & $\boldsymbol{R}_{\text {ac }}(\mathbf{\Omega})$ \\
\hline Pair 1 & 40 & 4.81 & 350 & 0.450 \\
\hline Pair 2 & 50 & 6.67 & 250 & 0.607 \\
\hline Pair 3 & 63 & 9.28 & 178 & 0.820 \\
\hline
\end{tabular}

TABLE II. CORRESPONDING TRANSMITTING DISTANCE OF EACH

PAIR FOR A SAME EFFICIENCY COEFFICIENT.
\begin{tabular}{|c|c|c|c|c|c|}
\hline $\mathbf{k}_{\mathbf{Q}}$ & 9 & 5 & 3 & 2 & 1 \\
\hline Pair 1 (mm) & 37 & 51 & 64 & 77 & 102 \\
\hline Pair 2 (mm) & 47 & 64 & 81 & 96 & 127 \\
\hline Pair 3 (mm) & 61 & 81 & 102 & 121 & 159 \\
\hline
\end{tabular}

Changing transmitting distance of the each pair according to Table II and choosing the corresponding optimal equivalent load resistance $R_{\mathrm{o} \_ \text {opt }}$ to maximize the efficiency of each pair, then tested maximum efficiency of different pairs under different efficiency coefficient $k_{\mathrm{Q}}$ are shown in Fig. 4.

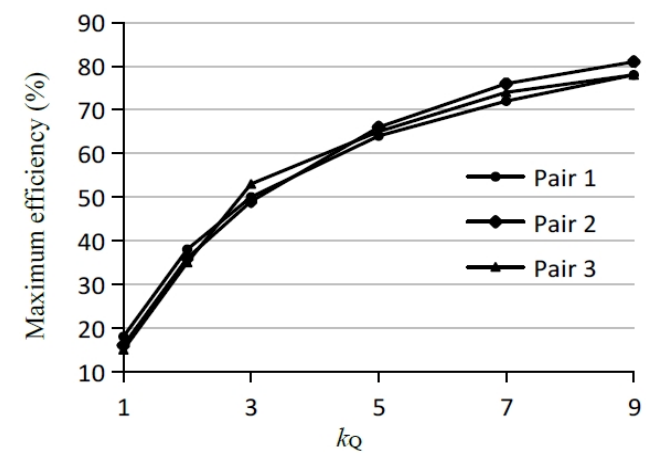

Fig. 4. Maximum efficiency of different pairs of windings vs. $k$ Q.

\section{DESIGN WITH ORTHOGONAL EXPERIMENTS METHOD}

\section{A. Parameters Definition for Orthogonal Experiments}

Using orthogonal experiment designing methods, the three parameters named with index, factors, and levels should be defined [16]. In the optimal design of the PCB windings with the four-winding structure as shown in Fig. 5, the index is defined with $k_{\mathrm{Q}}$.

It can be seen that the maximum efficiency of the different pairs will keep the same as long as their efficiency coefficient is the same, i.e. the maximum efficiency only depends on $k_{\mathrm{Q}}$. Moreover, the maximum efficiency increases with $k_{\mathrm{Q}}$. It should be noted that small difference (maximum difference is about $5 \%$ ) of the tested maximum efficiencies for a same $k_{\mathrm{Q}}$ is caused by testing error and parasitic parameters, such as self-capacitance of the windings etc.

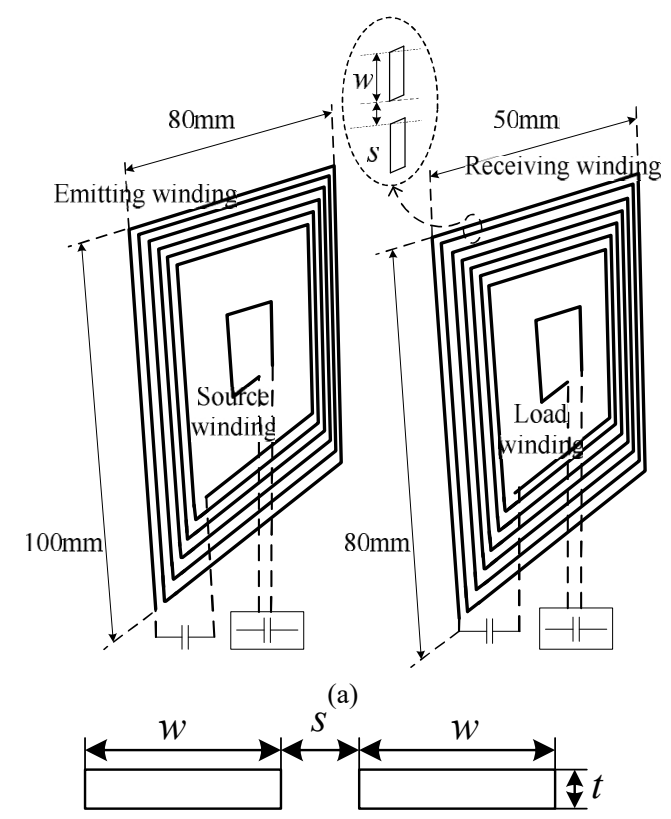

(b)

Fig. 5. Winding structure: a) Four-winding structure with PCB winding; b) PCB trace.

The factors are defined with geometrical parameters of the windings, including winding's number of turns $N$, the trace width $w$, the distance between adjacent traces $s$, and the trace thickness $t$. The levels are defined with the value of the factors or the geometrical parameters such as the $s$ can be given three different values with $0.5 \mathrm{~mm}, 1 \mathrm{~mm}$ and $1.5 \mathrm{~mm}$, respectively.

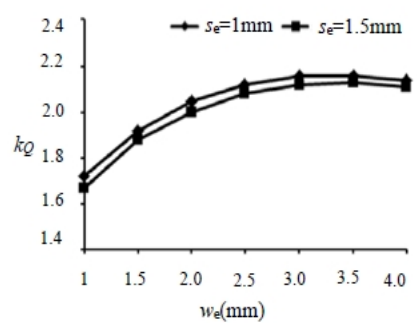

(a)

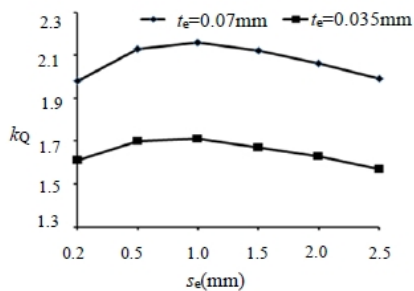

(d)

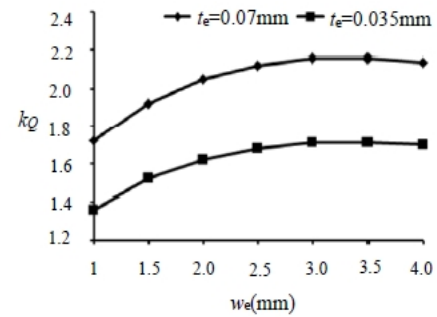

(b)

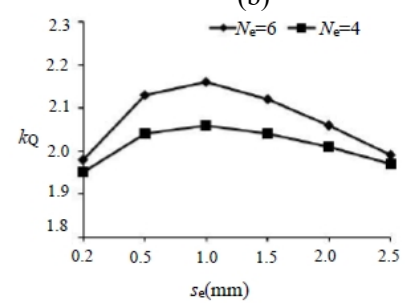

(e)

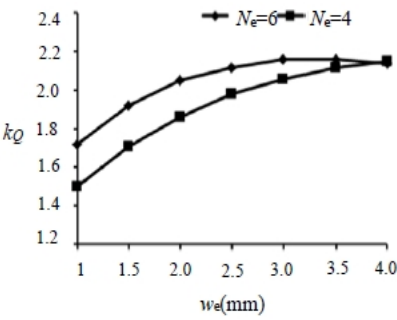

(c)

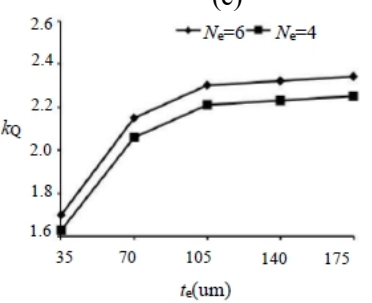

(f)

Fig. 6. Interactions analysis among the parameters or factors $N_{\mathrm{e}}, w_{\mathrm{e}}, s_{\mathrm{e}}$, and $t_{\mathrm{e}}$ : a) $k_{\mathrm{Q}} v_{s} . w_{\mathrm{e}}$ and $s_{\mathrm{e}}$; b) $k_{\mathrm{Q}} v s . w_{\mathrm{e}}$ and $t_{\mathrm{e}}$; c) $k_{\mathrm{Q}} v_{s} . w_{\mathrm{e}}$ and $N_{\mathrm{e}}$; d) $k_{\mathrm{Q}} v_{s} . s_{\mathrm{e}}$ and $t_{\mathrm{e}}$; e) $k_{\mathrm{Q}} v s . s_{\mathrm{e}}$ and $\left.N_{\mathrm{e}} ; \mathrm{f}\right) k_{\mathrm{Q}} v s . t_{\mathrm{e}}$ and $N_{\mathrm{e}}$ 
In the optimal design of the PCB windings, the constraints are maximum size of the emitting winding with width $80 \mathrm{~mm}$ and length $100 \mathrm{~mm}$ and the receiving winding with width $50 \mathrm{~mm}$ and length $80 \mathrm{~mm}$. And the transmitting distance is fixed to $130 \mathrm{~mm}$; source frequency is $4 \mathrm{MHz}$.

\section{B. Interaction Analysis of the Factors}

The factors include the winding geometrical parameters of $N_{\mathrm{e}}, w_{\mathrm{e}}, s_{\mathrm{e}}$, and $t_{\mathrm{e}}$ for the emitting winding. Here the subscript represents the parameters for the emitting winding. In order to get the orthogonal chart for orthogonal experiments, the interactions among the parameters is analysed. Using the eddy current field simulator from the FEM software Ansoft, the factor interactions of the emitting winding can be obtained as plotted in Fig. 6. In accordance with the judgment of orthogonal experiments and Fig. 6, it can be found that there are no or weak interactions between $w_{\mathrm{e}}$ and $s_{\mathrm{e}}, w_{\mathrm{e}}$ and $t_{\mathrm{e}}, s_{\mathrm{e}}$ and $t_{\mathrm{e}}, s_{\mathrm{e}}$ and $N_{\mathrm{e}}, t_{\mathrm{e}}$ and $N_{\mathrm{e}}$, but there exists strong interactions between $N_{\mathrm{e}}$ and $w_{\mathrm{e}}$. The same analysis and conclusions can be made to the receiving winding.

\section{Orthogonal Experiment Design}

In initial designs, given that $t_{\mathrm{e}}$ has three levels with $1 \mathrm{oz}$ $(1 \mathrm{oz}=35 \mathrm{um}), 2 \mathrm{oz}$, and $3 \mathrm{oz}$, Fig. 6 shows that $t_{\mathrm{e}}$ has no interaction with the other factors $N_{\mathrm{e}}, w_{\mathrm{e}}$ and $s_{\mathrm{e}}$, and Fig. 6(f) also shows that $k_{\mathrm{Q}}$ increases slightly with increasing of $t_{\mathrm{e}}$ at above $2 \mathrm{oz}$, while expense will increase greatly. Then $t_{\mathrm{e}}$ is fixed as $2 \mathrm{oz}$ in the whole design, i.e $t_{\mathrm{e}}$ has only one level. So $t_{\mathrm{e}}$ can be excluded from the factors in the design of orthogonal chart, resulting in reducing numbers of experiments. There are 6 factors (including $N_{\mathrm{e}}, w_{\mathrm{e}}, s_{\mathrm{e}}$ for the emitting winding and $N_{\mathrm{r}}, w_{\mathrm{r}}, S_{\mathrm{r}}$ for the receiving winding) and the each factor has 3 levels as given in Table III.

Considering the interaction factors $\left(N_{\mathrm{e}}\right.$ with $w_{\mathrm{e}}$, and $N_{\mathrm{r}}$ with $\left.w_{\mathrm{r}}\right)$, the orthogonal chart $L_{27}\left(3^{13}\right)$ is designed as Table IV. Table IV shows that there are 27 representative experiments totally; Except for the experiment number $n$, the “1", '2"'and '3" represent 'level 1', 'level 2' and 'level 3' of each factor as listed in Table III; The order of factors is determined by referring to [16] (" $\times$ " is the notation of interaction). The index of the calculated " $k_{Q}$ " is listed in the Table IV for each representative experiment by substituting $\mathrm{AC}$ resistance and mutual inductance of the windings obtained by FEM simulations into (4).

TABLE III. LEVELS CHART OF FACTORS

\begin{tabular}{|c|c|c|c|}
\hline & level 1 & level 2 & level 3 \\
\hline$N_{\mathrm{e}}($ turns) & 6 & 7 & 5 \\
\hline $\boldsymbol{N}_{\mathbf{r}}($ turns) & 6 & 4 & 5 \\
\hline $\boldsymbol{w}_{\mathrm{e}}(\mathbf{m m})$ & 3.5 & 2.5 & 3 \\
\hline $\boldsymbol{w}_{\mathbf{r}}(\mathbf{m m})$ & 3 & 2 & 2.5 \\
\hline $\boldsymbol{s}_{\mathrm{e}}(\mathbf{m m})$ & 1.5 & 0.5 & 1 \\
\hline $\boldsymbol{s}_{\mathbf{r}}(\mathbf{m m})$ & 0.2 & 0.5 & 1 \\
\hline
\end{tabular}

TABLE IV. $L_{27}\left(3^{13}\right)$ ORTHOGONAL CHART.

\begin{tabular}{|c|c|c|c|c|c|c|c|c|c|c|c|c|c|c|c|}
\hline \multirow{2}{*}{\multicolumn{2}{|c|}{ Level }} & \multicolumn{13}{|c|}{ Factors } & \multirow{3}{*}{$\frac{\mathbf{k}_{\mathbf{Q}}}{1.86}$} \\
\hline & & \multirow{2}{*}{$\begin{array}{c}N_{\mathrm{e}} \\
1\end{array}$} & \multirow{2}{*}{$\begin{array}{c}N_{\mathbf{r}} \\
1 \\
\end{array}$} & \multirow{2}{*}{$\begin{array}{c}\boldsymbol{s}_{\mathbf{e}} \\
1 \\
\end{array}$} & \multirow{2}{*}{$\begin{array}{c}\boldsymbol{s}_{\mathbf{r}} \\
1 \\
\end{array}$} & \multirow{2}{*}{$\begin{array}{c}w_{\mathrm{e}} \\
1\end{array}$} & \multirow{2}{*}{$\begin{array}{c}\boldsymbol{N} \times \boldsymbol{w} \\
1\end{array}$} & \multirow{2}{*}{$\begin{array}{c}N_{\mathrm{e}} \times w_{\mathrm{e}} \\
1\end{array}$} & \multirow{2}{*}{$\begin{array}{c}\text { null } \\
1 \\
\end{array}$} & \multirow{2}{*}{$\begin{array}{c}w_{\mathrm{r}} \\
1\end{array}$} & \multirow{2}{*}{$\begin{array}{c}\text { null } \\
1\end{array}$} & \multirow{2}{*}{$\begin{array}{c}\text { null } \\
1\end{array}$} & \multirow{2}{*}{$\begin{array}{c}N_{\mathbf{r}} \times w_{\mathbf{r}} \\
1\end{array}$} & \multirow{2}{*}{$\begin{array}{c}\text { null } \\
1\end{array}$} & \\
\hline \multirow{27}{*}{$n$} & 1 & & & & & & & & & & & & & & \\
\hline & 2 & 1 & 1 & 1 & 1 & 2 & 2 & 2 & 2 & 2 & 2 & 2 & 2 & 2 & 1.99 \\
\hline & 3 & 1 & 1 & 1 & 1 & 3 & 3 & 3 & 3 & 3 & 3 & 3 & 3 & 3 & 1.97 \\
\hline & 4 & 1 & 2 & 2 & 2 & 1 & 1 & 1 & 2 & 2 & 2 & 3 & 3 & 3 & 2.06 \\
\hline & 5 & 1 & 2 & 2 & 2 & 2 & 2 & 2 & 3 & 3 & 3 & 1 & 1 & 1 & 2.11 \\
\hline & 6 & 1 & 2 & 2 & 2 & 3 & 3 & 3 & 1 & 1 & 1 & 2 & 2 & 2 & 2.16 \\
\hline & 7 & 1 & 3 & 3 & 3 & 1 & 1 & 1 & 3 & 3 & 3 & 2 & 2 & 2 & 2.15 \\
\hline & 8 & 1 & 3 & 3 & 3 & 2 & 2 & 2 & 1 & 1 & 1 & 3 & 3 & 3 & 2.08 \\
\hline & 9 & 1 & 3 & 3 & 3 & 3 & 3 & 3 & 2 & 2 & 2 & 1 & 1 & 1 & 2.12 \\
\hline & 10 & 2 & 1 & 2 & 3 & 1 & 2 & 3 & 1 & 2 & 3 & 1 & 2 & 3 & 2.04 \\
\hline & 11 & 2 & 1 & 2 & 3 & 2 & 3 & 1 & 2 & 3 & 1 & 2 & 3 & 1 & 2.05 \\
\hline & 12 & 2 & 1 & 2 & 3 & 3 & 1 & 2 & 3 & 1 & 2 & 3 & 1 & 2 & 1.91 \\
\hline & 13 & 2 & 2 & 3 & 1 & 1 & 2 & 3 & 2 & 3 & 1 & 3 & 1 & 2 & 2.05 \\
\hline & 14 & 2 & 2 & 3 & 1 & 2 & 3 & 1 & 3 & 1 & 2 & 1 & 2 & 3 & 2.09 \\
\hline & 15 & 2 & 2 & 3 & 1 & 3 & 1 & 2 & 1 & 2 & 3 & 2 & 3 & 1 & 2.03 \\
\hline & 16 & 2 & 3 & 1 & 2 & 1 & 2 & 3 & 3 & 1 & 2 & 2 & 3 & 1 & 1.95 \\
\hline & 17 & 2 & 3 & 1 & 2 & 2 & 3 & 1 & 1 & 2 & 3 & 3 & 1 & 2 & 2.08 \\
\hline & 18 & 2 & 3 & 1 & 2 & 3 & 1 & 2 & 2 & 3 & 1 & 1 & 2 & 3 & 2.11 \\
\hline & 19 & 3 & 1 & 3 & 2 & 1 & 3 & 2 & 1 & 3 & 2 & 1 & 3 & 2 & 2.13 \\
\hline & 20 & 3 & 1 & 3 & 2 & 2 & 1 & 3 & 2 & 1 & 3 & 2 & 1 & 3 & 1.90 \\
\hline & 21 & 3 & 1 & 3 & 2 & 3 & 2 & 1 & 3 & 2 & 1 & 3 & 2 & 1 & 2.15 \\
\hline & 22 & 3 & 2 & 1 & 3 & 1 & 3 & 2 & 2 & 1 & 3 & 3 & 2 & 1 & 2.16 \\
\hline & 23 & 3 & 2 & 1 & 3 & 2 & 1 & 3 & 3 & 2 & 1 & 1 & 3 & 2 & 1.97 \\
\hline & 24 & 3 & 2 & 1 & 3 & 3 & 2 & 1 & 1 & 3 & 2 & 2 & 1 & 3 & 2.09 \\
\hline & 25 & 3 & 3 & 2 & 1 & 1 & 3 & 2 & 3 & 2 & 1 & 2 & 1 & 3 & 2.05 \\
\hline & 26 & 3 & 3 & 2 & 1 & 2 & 1 & 3 & 1 & 3 & 2 & 3 & 2 & 1 & 1.97 \\
\hline & 27 & 3 & 3 & 2 & 1 & 3 & 2 & 1 & 2 & 1 & 3 & 1 & 3 & 2 & 1.98 \\
\hline \multicolumn{2}{|c|}{$K_{1}$} & 18.49 & 18.00 & 18.18 & 18.00 & 18.45 & 17.95 & 18.51 & 18.44 & 18.08 & 18.46 & 18.40 & 18.17 & 18.39 & \\
\hline \multicolumn{2}{|c|}{$K_{2}$} & 18.30 & 18.71 & 18.32 & 18.64 & 18.23 & 18.45 & 18.55 & 18.41 & 18.48 & 18.32 & 18.36 & 18.81 & 18.41 & \\
\hline \multicolumn{2}{|c|}{$K_{3}$} & 18.39 & 18.49 & 18.69 & 18.55 & 18.51 & 18.80 & 18.13 & 18.34 & 18.63 & 18.41 & 18.44 & 18.22 & 18.39 & \\
\hline \multicolumn{2}{|c|}{$R$} & 0.19 & 0.71 & 0.51 & 0.64 & 0.28 & 0.85 & 0.42 & 0.09 & 0.55 & 0.14 & 0.08 & 0.64 & 0.03 & \\
\hline
\end{tabular}

Notes: $n$ means the number of experiments. $K_{i}$ is relative index, and $R$ is the range of the factor in the same column. 
According to the design of orthogonal experiments with analysis of $K_{1}, K_{2}, K_{3}$, and $R\left(K_{1}, K_{2}, K_{3}\right.$ are relative index, and $R$ is the range of the factor in the same column), the optimal PCB windings can be got as follows, $N_{\mathrm{e}}=5, N_{\mathrm{r}}=4, s_{\mathrm{e}}$ $=1 \mathrm{~mm}, s_{\mathrm{r}}=0.5 \mathrm{~mm}, w_{\mathrm{e}}=3.5 \mathrm{~mm}, w_{\mathrm{r}}=3 \mathrm{~mm}, t_{\mathrm{e}}=t_{\mathrm{r}}=0.07$ $\mathrm{mm}$. While the optimal design with the control variables of [15] is $N_{\mathrm{e}}=6, N_{\mathrm{r}}=5, s_{\mathrm{e}}=1 \mathrm{~mm}, \mathrm{~s}_{\mathrm{r}}=0.5 \mathrm{~mm}, w_{\mathrm{e}}=3 \mathrm{~mm}, w_{\mathrm{r}}=$ $2 \mathrm{~mm}, t_{\mathrm{e}}=t_{\mathrm{r}}=0.07 \mathrm{~mm}$.

\section{EXPERIMENTAL TEST}

In order to verify the theoretical analysis above, the PCB windings prototypes based on the method in [15] and the proposed orthogonal experiment designing method are constructed and thereby illustrated in Fig. 7(a) and Fig. 7(b), respectively.

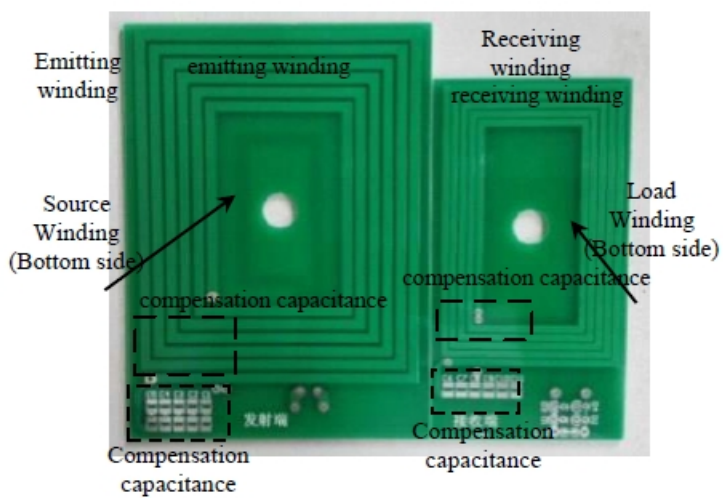

(a)

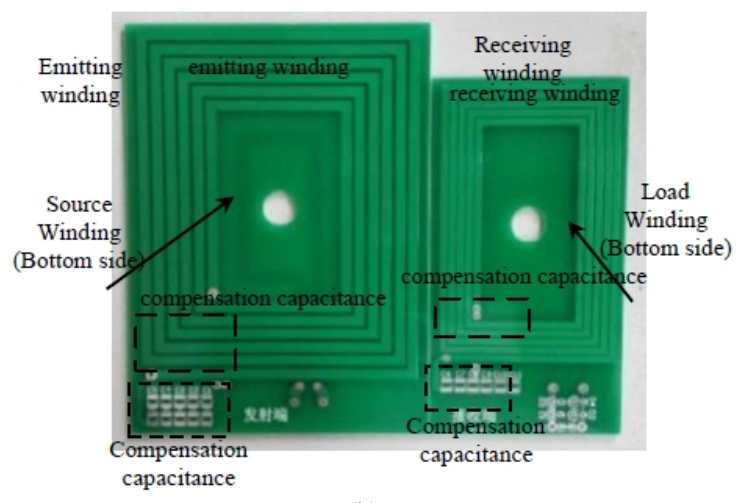

(b)

Fig. 7. Constructed windings prototypes: a) Windings optimized by method in [15]; b) Windings optimized by the orthogonal experiments.

The winding parameters $\mathrm{AC}$ resistance $R_{\mathrm{ac}}$ and mutual inductance $M$ measured by Impedance Analyzer WK-6500B at $4 \mathrm{MHz}$ frequency are given in Table $\mathrm{V}$ and Table VI.

TABLE V. PARAMETERS OF WINDINGS OPTIMIZED BY METHOD IN [15].

\begin{tabular}{|c|c|c|c|}
\hline Frequency at $4 \mathbf{M H z}$ & $R_{\mathrm{ac}}$ & $M$ & $k_{\mathrm{Q}}$ \\
\hline Emitting winding & $361 \mathrm{~m} \Omega$ & \multirow{2}{*}{$30.7 \mathrm{nH}$} & 2.19 \\
\hline Receiving winding & $343 \mathrm{~m} \Omega$ & & \\
\hline
\end{tabular}

TABLE VI. PARAMETERS OF WINDINGS OPTIMIZED BY ORTHOGONAL EXPERIMENTS.

\begin{tabular}{|c|c|c|c|}
\hline Frequency at $4 \mathbf{M H z}$ & $R_{\mathrm{ac}}$ & $M$ & $k_{\mathrm{Q}}$ \\
\hline Emitting winding & $255 \mathrm{~m} \Omega$ & \multirow{2}{*}{$21.0 \mathrm{nH}$} & 2.46 \\
\hline Receiving winding & $181 \mathrm{~m} \Omega$ & & \\
\hline
\end{tabular}

It shows that $k_{\mathrm{Q}}$ from (4) with the test winding parameter is increased by about $12 \%$. Then, the maximum efficiency from (3) is $41.3 \%$ and $45.2 \%$ respectively, and the efficiency increases by about $4 \%$.

\section{CONCLUSIONS}

The CMRU is a key component of the MR-WPT, and its maximum transmitting efficiency only depends on the $k_{\mathrm{Q}}$ determined by the intrinsic parameters, i.e. the windings coupled coefficient and its quality factor. The efficiency will decrease with decreasing $k_{\mathrm{Q}}$, and drops rapidly before the key $k_{\mathrm{Q}}$. The introducing of $k_{\mathrm{Q}}$ as design objective of the CMRU aims to the maximum efficiency. And in order to improve the efficiency, the $k_{\mathrm{Q}}$ must be optimized as large as possible by the windings geometry.

The proposed design method with orthogonal experiment and FEM simulation is for optimizing the coupled windings, especially the air-core PCB winding characterized of much more fringing magnetic field, excited by high-frequency source which is up to several hundreds of $\mathrm{kHz}$. In the method, the $k_{\mathrm{Q}}$ is defined to be as the index for optimizing objective, and the factors includes the winding turn numbers, the PCB trace width, and the distance between adjacent traces. But there only exists interaction between the winding turn numbers and the trace width, resulting in the small numbers of experiments.

The proposed design method not only optimizes the PCB windings to get high $k_{\mathrm{Q}}$ effectively, but also reduces the numbers of experiments greatly. The PCB winding design example shows that the $k_{\mathrm{Q}}$ was optimized to be increased by about $12 \%$, resulting in improving the maximum efficiency by about $4 \%$. The experiments and FEM simulation verified the analysis and the design method.

\section{REFERENCES}

[1] A. Kurs, et al, "Wireless energy transfer via strongly coupled magnetic resonances", Science, vol. 317, pp. 83-85, 2007. [Online]. Available: http://dx.doi.org/10.1126/science.1143254

[2] Zhao Zhengming, Zhang Yiming, Chen Kainan, "New progress of magnetically-coupled resonant wireless power transfer technology", in Proc. CSEE., 2013, pp. 1-13. [Online]. Available: http://dx.doi.org/ 10.13334/j.0258-8013.pcsee.2013.03.003

[3] S. Hui, W. Zhong, C. Lee, "A critical review of recent progress in mid-range wireless power transfer", IEEE Trans. on Power Electronics, vol. PP, pp. 1-1, 2013. [Online]. Available: http://dx.doi.org/10.1109/TPEL.2013.2249670

[4] U. K. Madawala, M. Neath, D. J. Thrimawithana, “A power-frequency controller for bidirectional inductive power transfer systems", IEEE Trans. Industrial Electronics, vol. 60, pp. 310-317, 2013. [Online] Available: http://dx.doi.org/10.1109/TIE.2011.2174537

[5] Dac-Binh Ha, Duc-Dung Tran, Vu Tran-Ha, Een-Kee Hong, "Performance of amplify-and-forward relaying with wireless power transfer over dissimilar channels", Elektronika ir Elektrotechnika, vol. 21, no. 5, pp. 91-95, 2015. [Online]. Available: http://dx.doi.org/ $10.1109 / 63.750181$

[6] S. Cheon, Y. H. Kim, S. Y. Kang, et al, "Circuit-model-based analysis of a wireless energy-transfer system via coupled magnetic resonances", IEEE Trans I.E, vol. 58, no. 7, pp. 2906-2914, 2011. [Online]. Available: http://dx.doi.org/10.1109/TIE.2010.2072893

[7] Hongchang Li, Kangping Wang, Lang Huang, et al "Dynamic modeling based on coupled modes for wireless power transfer systems", IEEE Trans on P.E, vol. 30, no. 11, pp. 6245-6253, 2015. [Online]. Available: http://dx.doi.org/10.1109/TPEL.2014.2376474

[8] J. A. Ferreira, "Improved analytical modeling of conductive losses in magnetic components", IEEE Trans. P. E., vol. 9, no. 1, pp. 127-131, 
1994. [Online]. Available: http://dx.doi.org/10.1109/63.285503

[9] R. Sullivan Charles, "Optimal choice for number of strands in a litz-wire transformer winding", IEEE Trans. P. E., vol. 14, no. 2, pp. 283-291, 1999. [Online]. Available: http://dx.doi.org/10.1109/ 63.750181

[10] R. P. Wojda, M. K. Kazimierczuk, "Analytical optimization of solid-round-wire windings", IEEE Trans. on I.E, vol. 60 , no. 3, pp. 1033-1041, 2013. [Online]. Available: http://dx.doi.org/ 10.1109/TIE.2012.2189543

[11] I. Lope, C. Carretero, J. Acero, et al, "AC power losses model for planar windings with rectangular cross-sectional conductors", IEEE Trans. P.E, vol. 29, no. 1, pp. 23-28, 2014. [Online]. Available: http://dx.doi.org/10.1109/TPEL.2013.2256928

[12] Z. Pantic, S Lukic, "Computationally-efficient, generalized expressions for the proximity-effect in multi-layer, multi-turn tubular coils for wireless power transfer systems", IEEE Trans. Magnetics, vol. 49, no. 11, pp. 5404-5416, 2013. [Online]. Available:
http://dx.doi.org/10.1109/TMAG.2013.2264486

[13] Z. Wang, X. Z. Wei, H. F. Dai, "Nested three-layer optimization method for magnetic coils used in $3 \mathrm{~kW}$ vehicle-mounted wireless power transfer system", IET Power Electronics, vol. 9, no. 13, pp. 2562-2570, 2016. [Online]. Available: http://dx.doi.org/10.1049/ iet-pel.2015.0840

[14] S. R. Cove, M. Ordonez, "Wireless-power-transfer planar spiral winding design applying track width ratio", IEEE Trans. I. A, vol. 51, no. 3, pp. 2423-2433, 2015. [Online]. Available: http://dx.doi.org/ 10.1109/TIA.2014.2372092

[15] H. Jiang, S. X. Liang, J. M. Zhang, et al, "Design and optimization of printed spiral coils in wireless power transfer for biomedical implants", 4th Int. Conf. Biomedical Engineering and Informatics, 2011, pp. 1064-1067. [Online]. Available: http://dx.doi.org/10.1109 /BMEI.2011.6098398

[16] L. Feibao, L. Shulong, X. Meiyu, L. Wenli, Methods of applied statistics. Beijing: Peking University Press, 2010, pp. 164-187. 